\title{
Breast Lesion Elastography Region of Interest Selection and Quantitative Heterogeneity: A Systematic Review and Meta-Analysis
}

\author{
HANAA A. ABDELHAMEED, M.D.; KHALED A. ALY, M.D. and NOHA H. SAKR, M.Sc. \\ The Department of Radiodiagnosis, Faculty of Medicine, Ain Shams University
}

\begin{abstract}
Background: Breast cancer is one of the most common causes of death among women worldwide. Early detection and diagnosis will be helpful to reduce mortality and improve prognosis. It is urgent to develop efficient detection technology for breast cancer. Mammographic screening is a valuable tool for early detection of breast cancer. However, the increased density of breast tissue significantly reduces the diagnostic accuracy.
\end{abstract}

Aim of Study: To provide an overview of the different reported elasticities of specific breast pathologies based on ultrasound elastography.

Material and Methods: A total of 35 articles including 8316 patients and 9057 breast lesions were included in the pooled analysis of which 3060 malignant lesions were included from 40 studies. The median incidence of malignant breast lesion is $37.1 \%$ calculated from the incidence of malignant lesions of all included studies. Seven of the included studies assessed VTIQ. Mean age varied along all included studies.

Results: The sensitivity and specificity of Emax, Emean and Eratio for the diagnosis of breast cancer varied according to the interpretative criteria used to define a test as positive. The summary estimates of sensitivity and specificity were $82.58 \%$ (95\% CI $78.32 \%$ to $86.16 \%$ ) and $84.12 \%$ (95\% CI $79.07 \%$ to $87.07 \%$ ) for Emean, $86.19 \%$ (95\% CI $81.60 \%$ to $89.77 \%$ ) and $88.56 \%$ (95\% CI $88.56 \%$ to $91.54 \%$ ) for Emax, and $87.50 \%$ (95\% CI $77.47 \%$ to $93.44 \%$ ) and $79.30 \%$ (95\% CI $68.21 \%$ to $87.24 \%$ ) for Eratio respectively. Regarding DOR, Emax achieved the highest value 48.32 (95\% CI 28.7 to 67.8) which means there are 48 times the odds of obtaining an Emax positive result in a diseased rather than a nondiseased person. Meta-regression analysis was conducted to assess the impact of two covaries; Emean and Emax using Likelihood ratio test and revealed significant difference existed with higher summary sensitivity $\left(X^{2}=35.04, p<001\right)$ and specificity $\left(X^{2}=18.65, p<001\right)$ in Emax than Emean. SROC curves were used to show the distribution of sensitivity and specificity of Emax, Emean and Eratio in the Roc space as well as the prediction region.

Conclusion: Our meta-analysis demonstrates that SWE is an accurate and reliable diagnostic tool in discriminating

Correspondence to: Dr. Hanaa A. Abdelhameed, The Department of Radiodiagnosis, Faculty of Medicine, Ain Shams University malignant and benign breast lesions. With wide application, SWE may significantly improve the early diagnostic of breast cancer. SWE can provide additional information on predicting breast cancer prognosis. However, the possibility of falsepositive and false-negative results should be considered during interpretation.

Key Words: Breast - Lesion - Elastography - Quantitative Heterogeneity.

\section{Introduction}

WITH costs and incidences of breast diseases ever increasing, improved methods of differential diagnosis based on quantitative measures of elasticity have been gaining support and interest for clinical utilization. Numerous studies have reported lower stiffness of benign masses compared to their relatively stiff and malignant counterparts, establishing a widely-accepted correlation between the measured elasticity of a mass and its pathology [1]

Shear Wave Elastography (SWE) is the most widely utilized clinical method of measuring invivo tissue elasticity. The traditional metrics of lesion elasticity from SWE include the mean, maximum and/or the relative elasticity of the lesion to the adjacent parenchyma (strain ratio) [2].

Each of these three measures has been evaluated for utility in improving the specificity of breast lesion diagnosis. Strain ratio has also demonstrated clinical utility in differential diagnosis [3], but combines the elasticities of the pathologic with healthy adjacent tissues. There is evidence to suggest that the pathology of the lesion also affects the mechanics of the surrounding tissues [4] and therefore the ratios of stiffness may not be optimal for stratification of malignancy risk. Mean and maximum measures of elastic modulus are generally useful in confirming cases with very high (malignant) or very low (benign) stiffness, but neither measure can consistently discern malignan- 
cy alone. Of these studied metrics, maximum elasticity has demonstrated the greatest promise in differential diagnosis and will be considered the metric against which new metrics should be evaluated [5]

Different studies have identified a wide range of thresholds for discriminating benign from malignant conditions-ranging from $50 \mathrm{kPa}[6]$ to $82.3 \mathrm{kPa}[7]$ based on the mean malignancy stiffness.

Although a growing corpus of literature encourages the inclusion of elasticity in clinical practice based on observed improvements in diagnostic specificity $[\mathbf{3 , 5 , 8 , 9}$, improved metrics and standardization are needed to facilitate the use of these technologies and to address the significant variability that confounds early clinical results [10] Lesion heterogeneity has been acknowledged as a potentially useful measure and has been assessed both qualitatively and semi-quantitatively [5] .

\section{Aim of the work:}

The purpose of this systematic review and metaanalysis was therefore to (I) Provide an overview of the different reported elasticities of specific breast pathologies based on ultrasound elastography, (II) Evaluate the relationship of ROI selection to the reported elasticity metrics and (III) Evaluate a new metric of elasticity heterogeneity to improve the discrimination between benign and malignant conditions.

\section{Material and Methods}

A- Criteria for considering studies for this review: I- Type of studies:

We included cross-sectional or Diagnostic Test Accuracy (DTA) observational researches examining both prospective and retrospective studies. Studies had to mention that all participants received a reference standard; investigators had to present cross-tabulated results of the index test and the reference standard ( $2 \times 2$ table), or had to report sufficient information to allow the $2 \times 2$ table data to be back-calculated. We excluded case reports and case series. All studies had to be conducted with approval by an Institutional Review Board (IRB) in which each patient provided informed consent.

\section{II- Participants:}

We included studies if assessed patients presented with breast lesions, provided quantitative measurements of mean and/or maximum elasticity from Shear-Wave Elastography (SWE), reported at least three replicates for each reported lesion type, which may include classification as benign, malignant or a specific pathology and provided a reference standard diagnosis in addition the elastographic measures. We excluded animal studies.

\section{III- Index tests:}

Eligible studies were that examined Shear-wave Elastography. We involved studies of variable technical approaches for SWE:

1- One-dimensional transient elastography (1 DSWE).

2- Point shear wave elastography (pSWE, former acoustic radiation force impulse elastography).

3- Two-dimensional shear wave elastography (2DSWE) such as virtual touch imaging quantification (VTIQ).

4- Three dimensional shear wave elastography. We also included studies comparing SWE and conventional ultrasound or combined approach versus the reference standard.

\section{IV- Target condition:}

Female patients presented with breast lesions (benign or malignant) for further investigation.

\section{V- Reference standards:}

For the aim of this review, we considered Breast Ultrasound is the gold standard. Studies that compared index test versus pathological biopsy (fine needle aspiration) were also eligible.

\section{$B$ - Search strategy:}

The articles were retrieved from Pubmed, Cochrane databases, Trip database, Google Scholar and different databases in Egyptian Knowledge Bank including; web of science, Elsevier, Science Direct, Scopus and Ovid database. The following search terms were used to retrieve articles: "Shear wave elastography," "SWE," "virtual touch tissue quantification," "VTTQ," and "breast," the references of retrieved articles were carefully checked for potential ones. Only the articles in English were considered. No restrictions in terms of publication date or status. Detailed search strategies and search terms were available in Appendix (1).

\section{C-Data collection and analysis:}

\section{I- Study selection:}

All search hits were first assessed by title and abstract for eligibility. Potentially eligible articles were examined in full text to decide on which studies to include or exclude.

PRISMA flow diagram illustrates the screening and study selection process. 


\section{II- Data extraction and management:}

We used the methods recommended by the Cochrane Screening and Diagnostic Test Methods Group. We developed a customised form to ensure reproducible collection of data items. Data collection was piloted on five manuscripts to ensure completeness of the form's items and to complement any missing items. We extracted the following per each study:

- Study design whether prospective or retrospective, country, mean age \pm SD (range), number of patients, number of lesions, number of benign le- sions, number of malignant lesions, reference standard used, technique used (SWE or VTIQ) and outcome parameters.

For the aim of this review and to calculate sensitivity and specificity, we extracted the number of true positive cases(sensitivity), False Negative cases (FN), False Positive cases (FP) and true negative cases (specificity) per each individual study Fig. (1).

Calculation of accuracy measures per each study:

\begin{tabular}{|c|c|c|c|}
\hline \multirow{2}{*}{$\begin{array}{l}\text { Test outcome } \\
\text { (index test) }\end{array}$} & \multicolumn{2}{|c|}{ Disease status (reference standard result) } & \multirow{2}{*}{ Total } \\
\hline & Diseased (D+) & Diseased (-) & \\
\hline Index test positive $(\mathrm{T}+)$ & True positives (a) & False positives (b) & Test positives $(a+b)$ \\
\hline Index test negative $(\mathrm{T}-)$ & False negatives (c) & True negatives (d) & Test negatives $(\mathrm{c}+\mathrm{d})$ \\
\hline Total & Disease positives $(a+c)$ & Disease positives $(b+d)$ & $N(a+b+c+d)$ \\
\hline
\end{tabular}

*: Adapted from Cochrane Handbook for DTA reviews.

We excluded studies that assessed the diagnostic performance of combined use of Elastography and Color Doppler US versus the reference standard where we couldn't retrieve the numeric data for elastography alone.

We attempted to contact the authors of included studies where information considered key to completion of a 2 X 2 table was unclear or missing.

\section{III- Statistical analysis and data synthesis:}

We performed statistical analysis as per the Cochrane guidelines for diagnostic test accuracy reviews (1). Two-by-two tables were constructed separately for Emax, Emean and Eratio where this information was available. We entered data from the individual studies into Review Manager 5 program (2) for exploratory analysis. We used reported data on test accuracy and disease prevalence or the True Positives (TP), True Negatives (TN), False Positives (FP), and False Negatives (FN), which ever was reported by the individual study. We calculated the sensitivity, specificity, and positive and negative likelihood ratios as well as measures of statistical uncertainty (e.g. 95\% confidence intervals). We presented data from each study graphically by plotting sensitivities and specificities on a coupled forest plot. Our metaanalysis was performed to allow for the trade-off and correlation between sensitivity and specificity that occurs between studies that vary in the threshold value used to define test positives and test negatives. We conducted statistical analysis using Bivariate random-effects model for meta-analysis due to concerns related to potential methodological and clinical heterogeneity across the included studies. We performed meta-analysis to enable studies to be combined that have used a test(s) at different thresholds so, we focused more on the interpretation of SROC curves per test rather than summary sensitivity and specificity (the average operating point). Summary ROC plots display the results of individual studies in ROC space, each study is plotted as a single sensitivity-specificity point. The size of points depicts the precision of the estimate (typically scaled according to the inverse of the standard error of the logit (sensitivity) and logit (specificity)) or according to their sample sizes. We compared the diagnostic accuracy of Emean and Emax using likelihood ratio test with restricting comparison to paired data only to avoid any potential bias related to clinical and methodological heterogeneity if studies assessed Emean and Emax independently. We calculated another measure of diagnostic accuracy; the Diagnostic Odds Ratio (DOR). It summarizes the diagnostic accuracy of the index test as a single number that describes how many times higher the odds are of obtaining a test positive result in a diseased rather than a non-diseased person.

Statistical analysis was performed using the glmer function in the lme4 package of R software (3).

\section{- Investigations of heterogeneity:}

The potential sources of heterogeneity included baseline prevalence of breast cancer in the target 
population, the cut off points used to determine a positive test result, the reference standard used to diagnose breast cancer and study design.

We visually inspected SROC curves for the degree to which the observed study results lied close to the summary ROC curve, not how scattered they are in ROC space.

The magnitude of observed heterogeneity is best depicted graphically where such relationships can be observed by the scatter of points and from the prediction ellipse.

Prediction region is one way of illustrating the extent of statistical heterogeneity by depicting a region within which, assuming the model is correct, we have $95 \%$ confidence that the true sensitivity and specificity of a future study should lie (3).

\section{- Sensitivity analyses:}

We conducted meta-regression analysis to explore source of heterogeneity by adding different elastography measures (Emean, Emax) as covariates in the model. likelihood ratio tests can be used to compare models with or without a covariate term.

\section{Results}

\section{A- Results of search:}

Our search initially identified 1198 papers from different sources (see PRISMA flow diagram). Duplicates were removed and we screened 715 articles by title and abstract then 601 papers were excluded. We obtained full-text copies of 114 papers, of which 53 were excluded after full text assessment. We finally included 35 articles in our analysis. In some articles, numeric data were unavailable, only combined elastography and U/S versus U/S comparison or outcomes were irrelevant after full text screening hence were excluded.

In total, 8316 women with 9057 breast lesions, of which 3060 malignant lesions were included from 40 studies. The median incidence of malignant breast lesion is $37.1 \%$ calculated from the incidence of malignant lesions of all included studies. Seven of the included studies assessed VTIQ. Mean age varied along all included studies. Table (1) fully illustrates the characteristics of included studies.

\section{B- Findings:}

The summary estimates of sensitivity and specificity were $82.58 \%(95 \%$ CI $78.32 \%$ to $86.16 \%)$ and $84.12 \%$ (95\% CI $79.07 \%$ to $87.07 \%$ ) for Emean, $86.19 \%$ (95\% CI $81.60 \%$ to $89.77 \%$ ) and $88.56 \%$ (95\% CI $88.56 \%$ to $91.54 \%$ ) for Emax, and $87.50 \%$ (95\% CI $77.47 \%$ to $93.44 \%$ ) and $79.30 \%$ (95\% CI $68.21 \%$ to $87.24 \%$ ) for Eratio respectively. LR+/LR- were 5.20 (95\% CI 3.67 to 6.74)/0.21 (95\% CI 0.16 to 0.26 ) for Emean, and 7.54 (95\% CI 5.37 to 9.70$) / 0.16$ (95\% CI 0.11 to 0.20 ) for Emax and 4.23 (95\% CI 2, 4 to 6.05)/0.16 (95\% CI 0.07 to 0.25 ) for Eratio.

Regarding DOR, Emax achieved the highest value 48.32 (95\% CI 28.7 to 67.8 ) which means There are 48 times the odds of obtaining an Emax positive result in a diseased rather than a nondiseased person.

Meta-regression analysis was conducted to assess the impact of two covaries; Emean and Emax using Likelihood ratio test and revealed significant difference existed with higher summary sensitivity $\left(\mathrm{X}^{2}=35.04, p<001\right)$ and specificity $(\mathrm{X}=$ $18.65, p<001)$ in Emax than Emean.

SROC curves shows the distribution of sensitivity and specificity of Emax, Emean and Eratio in the Roc space as well as the prediction region.

When used in isolation, Emax positive test as diagnostic criterion for breast cancer in a population of 100 people with a disease prevalence of $40 \%$ (overall mean prevalence, overestimated due to retrospective design in many included studies), eight people who have breast cancer would be missed (sensitivity $82.5 \%, 17.5 \%$ false negatives), and 10 people would be unnecessarily treated or referred for further tests (specificity of $84.12 \%$, $15.9 \%$ false positive).

\begin{tabular}{llllllllll} 
Table (1) & \multicolumn{7}{c}{ Emax } \\
\cline { 2 - 9 } & Estimate & $95 \%$ LCI & $95 \%$ UCI & Estimate & $95 \%$ LCI & $95 \%$ UCI & Estimate & $95 \%$ LCI & $95 \%$ UCI \\
\hline Sn & $82.58 \%$ & $78.32 \%$ & $86.16 \%$ & $86.19 \%$ & $81.60 \%$ & $89.77 \%$ & $87.50 \%$ & $77.47 \%$ & $93.44 \%$ \\
Sp & $84.12 \%$ & $79.07 \%$ & $87.07 \%$ & $88.56 \%$ & $84.71 \%$ & $91.54 \%$ & $79.30 \%$ & $68.21 \%$ & $87.24 \%$ \\
DOR & 25.13 & 13.49 & 36.76 & 48.32 & 28.74 & 67.89 & 26.82 & 7.71 & 45.93 \\
LR+ & 5.20 & 3.67 & 6.74 & 7.54 & 5.37 & 9.70 & 4.23 & 2.40 & 6.05 \\
LR- & 0.21 & 0.16 & 0.26 & 0.16 & 0.11 & 0.20 & 0.16 & 0.07 & 0.25 \\
\hline
\end{tabular}


Study

Au et al 2014

Bayat et al 2017

Cebi et al 2014

Chang et al 2013a

Chang et al 2013b

Chang et al $2013 \mathrm{c}$

Chang et al $2013 \mathrm{~d}$

Chang et al 20138

Chang et al 2017

Choi et al 2016

Choi et al 2018

Chung et al 2016

Harcos et al 2014

Klotz et al 2014

Lee et al 2013

Lee et al 2015

Lee et al_b 2013

Lin et al 2018

Moon et al 2017 (ROI-M)

Moon et al 2017(ROI-S)

Moon et al 2018

$\mathrm{Ng}$ et al 2016

Park et al 2015

Shi et al 2015

Shin et al 2017

Song et al 2018

Sun et al 2019

Tian et al 2017

Xiao et al 2014

Yoon et al 2013

Zhang et al 2015

Zhang et al 201 ?

$\begin{array}{rrrr}\text { TP } & \text { FP } & \text { FN } & \text { TN } \\ 40 & 9 & 4 & 70 \\ 95 & 14 & 13 & 105 \\ 31 & 5 & 1 & 78 \\ 71 & 39 & 0 & 40 \\ 70 & 27 & 1 & 52 \\ 68 & 12 & 3 & 67 \\ 57 & 11 & 14 & 68 \\ 30 & 3 & 41 & 76 \\ 27 & 11 & 6 & 90 \\ 58 & 2 & 16 & 40 \\ 6 & 13 & 2 & 35 \\ 54 & 8 & 7 & 10 \\ 38 & 12 & 15 & 74 \\ 95 & 8 & 7 & 57 \\ 59 & 8 & 8 & 69 \\ 26 & 3 & 4 & 107 \\ 32 & 27 & 4 & 93 \\ 638 & 225 & 114 & 1285 \\ 36 & 11 & 16 & 91 \\ 41 & 16 & 11 & 86 \\ 52 & 66 & 16 & 180 \\ 74 & 2 & 0 & 83 \\ 62 & 10 & 13 & 71 \\ 111 & 5 & 8 & 145 \\ 112 & 43 & 28 & 143 \\ 74 & 2 & 33 & 100 \\ 80 & 15 & 19 & 138 \\ 65 & 5 & 8 & 132 \\ 30 & 1 & 14 & 80 \\ 41 & 33 & 6 & 142 \\ 42 & 9 & 13 & 97 \\ 26 & 10 & 8 & 45\end{array}$

N Sensitivity $(95 \% \mathrm{Cl})$ Specificity $(95 \% \mathrm{Cl})$ $0.91[0.78,0.97]$

0.88 [0.80, 0.93] $0.97[0.84,1.00]$ $1.00[0.95,1.00]$ $0.99[0.92,1.00]$ $0.96[0.88,0.99]$ $0.80[0.69,0.89]$ $0.42[0.31,0.55]$ 0.82 [0.65, 0.93] $0.78[0.67,0.87]$ $0.75[0.35,0.97]$ $0.89[0.78,0.95]$ $0.72[0.58,0.83]$ $0.93[0.86,0.97]$ $0.88[0.78,0.95]$ $0.87[0.69,0.96]$ $0.89[0.74,0.97]$ $0.85[0.82,0.87]$ $0.69[0.55,0.81]$ $0.79[0.65,0.89]$ $0.76[0.65,0.86]$ $1.00[0.95,1.00]$ $0.83[0.72,0.90]$ $0.93[0.87,0.97]$ $0.80[0.72,0.86]$ $0.69[0.59,0.78]$ $0.81[0.72,0.88]$ $0.89[0.80,0.95]$ $0.68[0.52,0.81]$ $0.87[0.74,0.95]$

$0.76[0.63,0.87]$ $0.76[0.59,0.89]$

$0.89[0.79,0.95]$

0.88 [0.81, 0.93]

$0.94[0.86,0.98]$

$0.51[0.39,0.62]$

$0.66[0.54,0.76]$

$0.85[0.75,0.92]$

$0.86[0.76,0.93]$

$0.96[0.89,0.99]$

$0.89[0.81,0.94]$

$0.95[0.84,0.99$ ]

$0.73[0.58,0.85]$

$0.56[0.31,0.78]$

$0.86[0.77,0.93]$

$0.88[0.77,0.95]$

$0.90[0.81,0.95]$

$0.97[0.92,0.99]$

$0.78[0.69,0.85]$

$0.85[0.83,0.87]$

$0.89[0.82,0.94]$

$0.84[0.76,0.91]$

$0.73[0.67,0.79]$

$0.98[0.92,1.00]$

$0.88[0.78,0.94]$

$0.97[0.92,0.99]$

$0.77[0.70,0.83]$

$0.98[0.93,1.00]$

$0.90[0.84,0.94]$

$0.96[0.92,0.99]$

$0.99[0.93,1.00]$

$0.81[0.75,0.87]$

$0.92[0.84,0.96]$

$0.82[0.69,0.91]$

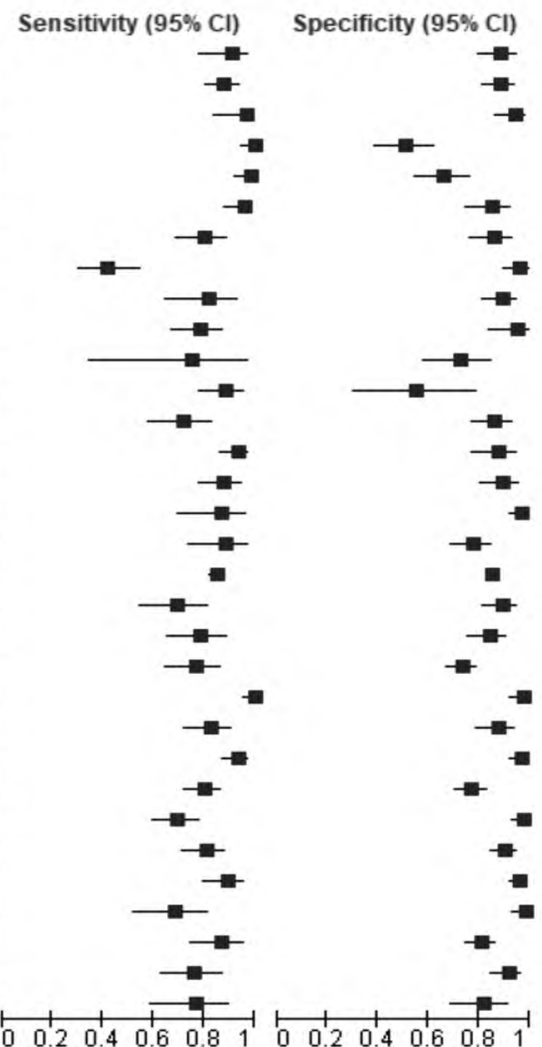

Forest plot of 2 Emax.

Study

Au et al 2014

Bayat et al 2017

Cebi et al 2014

Chang et al 2017

Choi et al 2016

Choi et al 2018

Chung et al 2016

Harcos et al 2014

Klotz et al 2014

Lee et al 2013

Lee et al_b 2013

Li et al 2016

Lin et al 2018

Moon et al 2017 (ROL-M)

Moon et al 2017(ROI-S)

Moon et al 2018

$\mathrm{Ng}$ et al 2016

Park et al 2015

Shi et al 2015

Shin et al 2017

Song et al 2018

Sun et al 2019

Xiao et al 2014

Zhang et al 2015

Zhang et al 2017

$\begin{array}{rrrrr}\text { TP } & \text { FP } & \text { FN } & \text { TN } & \text { Sensitivity }(95 \% \text { CI) } \\ 39 & 51 & 5 & 28 & 0.89[0.75,0.96] \\ 91 & 12 & 17 & 107 & 0.84[0.76,0.91] \\ 31 & 4 & 1 & 79 & 0.97[0.84,1.00] \\ 27 & 7 & 6 & 94 & 0.82[0.65,0.93] \\ 58 & 3 & 16 & 39 & 0.78[0.67,0.87] \\ 6 & 12 & 2 & 36 & 0.75[0.35,0.97] \\ 51 & 7 & 10 & 11 & 0.84[0.72,0.92] \\ 47 & 29 & 6 & 57 & 0.89[0.77,0.96] \\ 95 & 9 & 7 & 56 & 0.93[0.86,0.97] \\ 60 & 8 & 7 & 69 & 0.90[0.80,0.96] \\ 32 & 28 & 4 & 92 & 0.89[0.74,0.97] \\ 41 & 12 & 6 & 57 & 0.87[0.74,0.95] \\ 527 & 263 & 225 & 1247 & 0.70[0.67,0.73] \\ 33 & 3 & 19 & 89 & 0.63[0.49,0.76] \\ 42 & 19 & 10 & 83 & 0.81[0.67,0.90] \\ 50 & 72 & 18 & 174 & 0.74[0.61,0.83] \\ 74 & 3 & 0 & 82 & 1.00[0.95,1.00] \\ 60 & 10 & 15 & 71 & 0.80[0.69,0.88] \\ 106 & 24 & 23 & 126 & 0.82[0.74,0.88] \\ 111 & 47 & 29 & 139 & 0.79[0.72,0.86] \\ 87 & 18 & 30 & 84 & 0.74[0.65,0.82] \\ 78 & 12 & 21 & 141 & 0.79[0.69,0.86] \\ 30 & 16 & 14 & 65 & 0.68[0.52,0.81] \\ 38 & 24 & 17 & 82 & 0.69[0.55,0.81] \\ 25 & 9 & 9 & 46 & 0.74[0.56,0.87]\end{array}$

Specificity $(95 \% \mathrm{CI})$ $0.35[0.25,0.47]$ $0.90[0.83,0.95]$ $0.95[0.88,0.99]$ $0.93[0.86,0.97]$ $0.93[0.81,0.99]$ $0.75[0.60,0.86]$ $0.61[0.36,0.83]$ $0.66[0.55,0.76]$ $0.86[0.75,0.93]$ $0.90[0.81,0.95]$ $0.77[0.68,0.84]$ $0.83[0.72,0.91]$ $0.83[0.81,0.84]$ $0.97[0.91,0.99]$ $0.81[0.72,0.88]$ $0.71[0.65,0.76]$ $0.96[0.90,0.99]$ $0.88[0.78,0.94]$ $0.84[0.77,0.89]$ $0.75[0.68,0.81]$ $0.82[0.74,0.89]$ $0.92[0.87,0.96]$ $0.80[0.70,0.88]$ $0.77[0.68,0.85]$ $0.84[0.71,0.92]$

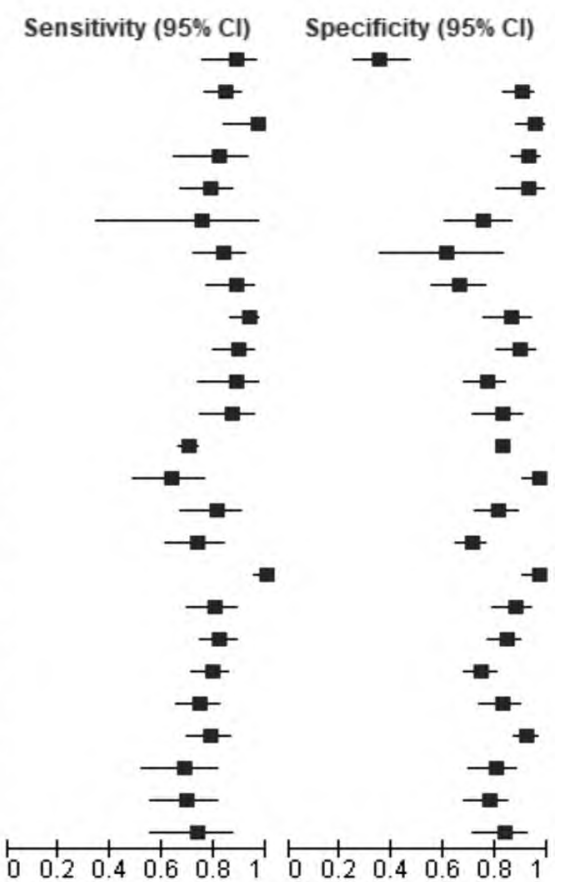

Forest plot of Emean. 
Emean

Study

Au et al 2014

Bayat et al 2017

Cebi et al 2014

Chang et al 2017

Choi et al 2016

Choi et al 2018

Chung et al 2016

Harcos et al 2014

Klotz et al 2014

Lee et al 2013

Lee et al_b 2013

Lin et al 2018

Moon et al 2017 (ROI-M)

Moon et al 2017 (ROI-S)

Moon et al 2018

$\mathrm{Ng}$ et al 2016

Park et al 2015

Shi et al 2015

Shin et al 2017

Song et al 2018

Sun et al 2019

Xiao et al 2014

Zhang et al 2015

Zhang et al 2017

Emax

Study

Au et al 2014

Bayat et al 2017

Cebi et al 2014

Chang et al 2017

Choi et al 2016

Choi et al 2018

Chung et al 2016

Harcos et al 2014

Klote et al 2014

Lee et al 2013

Lee et al_b 2013

Lin et al 2018

Moon et al 2017 (ROL-M)

Moon et al 2017(ROl-S)

Moon et al 2018

$\mathrm{Ng}$ et al 2016

Park et al 2015

Shi et al 2015

Shin et al 2017

Song et al 2018

Sun et al 2019

Xiao et al 2014

Zhang et al 2015

Zhang et al 2017

$\begin{array}{rrrrr}\text { TP } & \text { FP } & \text { FN } & \text { TN } & \text { Sensitivity }(95 \% \text { CI) } \\ 39 & 51 & 5 & 28 & 0.89[0.75,0.96] \\ 91 & 12 & 17 & 107 & 0.84[0.76,0.91] \\ 31 & 4 & 1 & 79 & 0.97[0.84,1.00] \\ 27 & 7 & 6 & 94 & 0.82[0.65,0.93] \\ 58 & 3 & 16 & 39 & 0.78[0.67,0.87] \\ 6 & 12 & 2 & 36 & 0.75[0.35,0.97] \\ 51 & 7 & 10 & 11 & 0.84[0.72,0.92] \\ 47 & 29 & 6 & 57 & 0.89[0.77,0.96] \\ 95 & 9 & 7 & 56 & 0.93[0.86,0.97] \\ 60 & 8 & 7 & 69 & 0.90[0.80,0.96] \\ 32 & 28 & 4 & 92 & 0.89[0.74,0.97] \\ 527 & 263 & 225 & 1247 & 0.70[0.67,0.73] \\ 33 & 3 & 19 & 89 & 0.63[0.49,0.76] \\ 42 & 19 & 10 & 83 & 0.81[0.67,0.90] \\ 50 & 72 & 18 & 174 & 0.74[0.61,0.83] \\ 74 & 3 & 0 & 82 & 1.00[0.95,1.00] \\ 60 & 10 & 15 & 71 & 0.80[0.69,0.88] \\ 106 & 24 & 23 & 126 & 0.82[0.74,0.88] \\ 111 & 47 & 29 & 139 & 0.79[0.72,0.86] \\ 87 & 18 & 30 & 84 & 0.74[0.65,0.82] \\ 78 & 12 & 21 & 141 & 0.79[0.69,0.86] \\ 30 & 16 & 14 & 65 & 0.68[0.52,0.81] \\ 38 & 24 & 17 & 82 & 0.69[0.55,0.81] \\ 25 & 9 & 9 & 46 & 0.74[0.56,0.87]\end{array}$

Specificity (95\% CI) $0.35[0.25,0.47]$

$0.90[0.83,0.95]$

$0.95[0.88,0.99]$

$0.93[0.86,0.97]$

$0.93[0.81,0.99]$

$0.75[0.60,0.86]$

$0.61[0.36,0.83]$

$0.66[0.55,0.76]$

$0.86[0.75,0.93]$

$0.90[0.81,0.95]$

$0.77[0.68,0.84]$

$0.83[0.81,0.84]$

$0.97[0.91,0.99]$

$0.81[0.72,0.88]$

$0.71[0.65,0.76]$

$0.96[0.90,0.99]$

$0.88[0.78,0.94]$

$0.84[0.77,0.89]$

$0.75[0.68,0.81]$

$0.82[0.74,0.89]$

$0.92[0.87,0.96]$

$0.80[0.70,0.88]$

$0.77[0.68,0.85]$

$0.84[0.71,0.92]$

0

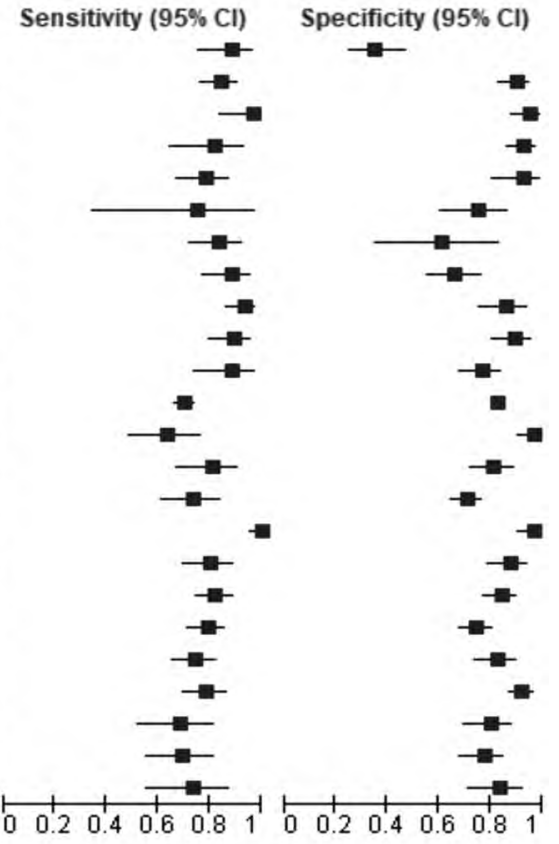
$0.89[0.79,0.95]$ $0.88[0.81,0.93$ ] $0.94[0.86,0.98]$ $0.89[0.81,0.94]$ $0.95[0.84,0.99]$ $0.73[0.58,0.85]$ $0.56[0.31,0.78]$ $0.86[0.77,0.93]$ $0.88[0.77,0.95]$ $0.90[0.81,0.95]$ $0.78[0.69,0.85]$ $0.85[0.83,0.87]$ $0.89[0.82,0.94]$ $0.84[0.76,0.91]$ $0.73[0.67,0.79]$ $0.98[0.92,1.00]$ $0.88[0.78,0.94]$ $0.97[0.92,0.99]$ $0.77[0.70,0.83]$ $0.98[0.93,1.00]$ $0.90[0.84,0.94]$ $0.99[0.93,1.00]$ $0.92[0.84,0.96]$ $0.82[0.69,0.91]$

Forest plot of for comparison of Emean and Emax (paired data only).

\section{Discussion}

Breast cancer is one of serious diseases threatening women's health. It is the major cause of death among women [11]. Moreover, the occurrence rate of this cancer has been increasing in recent years. Early detection and diagnosis will be helpful to reduce mortality and improve prognosis. It is urgent to develop efficient detection technology for breast cancer [12].
Shear Wave Elastography (SWE) is an emerging technique, which can obtain quantitative elasticity values in breast disease [13]. Recently, there has been great interest in the utilization of qualitative and quantitative information on breast lesions derived through breast elastography to distinguish between benign and malignant lesions. There exist several types of breast elastography, including strain imaging by compression, Acoustic Radiation Force Impulse (ARFI), and Shearwave Elastogra- 
phy (SWE). SWE produces additional quantitative values of tissue elasticity (in $\mathrm{kPa}$ units) and $\mathrm{SW}$ velocity (in sm/s units) [14]. This novel technology allows physician to obtain promising information, adjunctive to greyscale ultrasound, in differentiating benign from malignant breast masses [15]

The aim of work of the current study was to provide an overview of the different reported elasticities of specific breast pathologies based on ultrasound elastography, (ii) evaluate the relationship of ROI selection to the reported elasticity metrics and (iii) evaluate a new metric of elasticity heterogeneity to improve the discrimination between benign and malignant conditions.

Among the included studies, the application value of SWE in discriminating malignant and benign breast lesions was controversial. In the study of Zhang et al., [15] as 125 women included in their study with 171 lesions found that the sensitivity of SWE was only 0.76 and the specificity was 0.82 compared with other studies.

Cebi et al., [16] as 109 women with 115 breast lesions were included to analyze the diagnostic performance of SWE, Emax, Emean, and Emin were adopted to represent tissue stiffness. However, the diagnostic sensitivity (0.97) and specificity (0.94) of these parameters were high.

Also, Youk et al., [17] reported high detection sensitivity (0.92) and specificity (0.92) of SWE, in which Emax represent tissue elasticity.

Klotz et al., [18] found that the detection sensitivity of SWE was 0.93 (0.86-0.97), whereas specificity was 0.88 (0.77-0.95).

Au et al., [19] reported 0.91 detection sensitivity and 0.89 detection specificity. The variances in results might be attributed to the differences in characters of patients, ethnicity or SWE parameters.

But the sensitivity and specificity of Emax, Emean and Eratio for the diagnosis of breast cancer in the study done by $\mathrm{Ng}$ et al., [20] was $100 \%$ and 0.98 .

In a retrospective study, Chung and associates [21] evaluated the diagnostic performance of SWE for the differential diagnosis of breast papillary lesions. A total of 79 breast papillary lesions in 71 consecutive women underwent ultrasound and SWE prior to biopsy. Ultrasound features and quantitative SWE parameters were recorded for each lesion. All lesions were surgically excised or excised using an ultrasound-guided vacuumassisted method. The diagnostic performances of the quantitative SWE parameters were compared using the AUC. Of the 79 lesions, 6 (7.6\%) were malignant and $12(15.2 \%)$ were atypical. Orientation, margin, and the final BI-RADS ultrasound assessments were significantly different for the papillary lesions $(p<0.05)$. All qualitative SWE parameters were significantly different $(p<0.05)$. The AUC values for SWE parameters of benign and atypical or malignant papillary lesions ranged from 0.707 to 0.757 (sensitivity, 44.4 to $94.4 \%$; specificity, 42.6 to $88.5 \%$ ). The maximum elasticity and the mean elasticity showed the highest AUC (0.757) to differentiate papillary lesions. The authors concluded that SWE provided additional information for the differential diagnosis of breast papillary lesions; quantitative SWE features were helpful to differentiate breast papillary lesions. Moreover, they stated that further study about breast papillary lesions using larger ROI size his study had several drawbacks; (I) Small sample size $(\mathrm{n}=71)$. Of 217 papillary lesions diagnosed by USguided CNB, only 79 (36.4\%) were finally included due to the lack of a SWE image or no excision. Thus, large numbers and multi-center studies are needed in the future, (II) The authors did not compare their results with those of conventional US or assess combined diagnostic performance; further studies combining SWE and conventional US in are needed to differentiate papillary lesions, (III) These researchers used a 2 X 2-mm sized ROI. Larger ROI might be more accurate for the assessment of the breast masses by providing both maximum stiffness and heterogeneity of breast lesions.

In a systematic review and meta-analysis, Blank and colleagues [14] reported measured elasticity of benign and malignant breast pathologies from SWE, quantitatively confirmed the effect of the selected ROI on these measures and tested the hypothesis that a metric of heterogeneity based on the mean and maximum elasticity can improve specificity of diagnosis. The elasticity of benign, malignant and specific pathologic states were reported from 22 publications encompassing 2,989 patients, identified from a structured search of the literature from May to September 2015. A total of 12 articles were included in a meta-analysis that grouped results by the method of ROI selection to discriminate between different pathologies. These researchers observed a significant correlation between the method of selection of ROI for malignant mean $(p<0.001)$ and maximum $(p=0.027)$ elasticity, but no correlation with benign measures. They defined a quantitative heterogeneity parameter, the "stiffness gradient", computed from the mean and 
maximum measured elasticity. The stiffness gradient out-performed the current standard maximum elasticity metric in stratifying malignancy risk by a margin of $15 \%$ for the partial ROI, and $42 \%$ for the maximized ROI. An anecdotal example of improved differentiation using the stiffness gradient on pathology-specific lesions was also provided. The authors concluded that these results quantitatively indicated that the method of ROI selection in SWE not only has a significant impact on the resulting mean reported elasticity of a lesion, but may provide some insight into lesion heterogeneity. They stated that these findings suggested that further exploration of quantitative heterogeneity is needed to improve the specificity of diagnosis.

In a meta-analysis, Chen and colleagues [22] examined the performance of SWE for the differentiation of benign and malignant breast lesions. PubMed, Embase and the Cochrane library were searched for studies published up to January 2014. The references of retrieved relevant articles were reviewed to identify potential publications. Random-effect meta-analysis was conducted to assess the overall sensitivity and specificity of SWE in the differentiation of breast lesions. A total of 11 articles, including 2,424 patients, were included in the present meta-analysis. The summarized sensitivity and specificity of the SWE performance based on maximum elasticity were 0.93 (95\% CI: 0.9 to 0.95 ) and 0.81 (95\% CI: 0.78 to 0.83 ), respectively. For the mean elasticity, the summarized sensitivity and specificity were 0.94 (95\% CI: 0.92 to 0.96 ) and 0.71 (95\% CI: 0.69 to 0.74 ), respectively. The summarized sensitivity and specificity were 0.77 (95\% CI: 0.70 to 0.83 ) and 0.88 (95\% CI: 0.84 to 0.91 ) for the SD of elasticity. The authors concluded that SWE has a high sensitivity and specificity in the differentiation of benign and malignant breast lesions. However, they stated that more large and prospective studies are needed to further examine the performance of SWE.

In a meta-analysis, Liu et al., [23] estimated the diagnostic performance of SWE in differentiating malignant from benign breast lesions. A literature search of PubMed, Web of Science and Scopus up to November 2014 was conducted. A summary receiver operating characteristic curve was constructed, and pooled weighted estimates of sensitivity and specificity were calculated using a bivariate mixed-effects regression model. A total of 33 studies, which included a total of 5,838 lesions (2,093 malignant, 3,745 benign) from 5,397 patients, were finally analyzed. Summary sensitivity and specificity were 0.886 (95\% CI: 0.858 to 0.909 ) and 0.866 (95\% CI: 0.833 to 0.894$)$, respectively.
The pooled diagnostic OR was 50.41 (95\% CI: 34.972 to 72.664 ). And the area under the receiver operating characteristic curve of SWE was 0.94 (95\% CI: 0.91 to 0.96 ). No publication bias existed among these studies $(p=0.245)$. In the subgroup analysis, sensitivity and specificity were 0.862 (95\% CI: 0.811 to 0.901 ) and 0.875 (95\% CI: 0.793 to 0.928 ) among 1,552 lesions from 1,429 patients in the 12 studies using acoustic radiation force impulse imaging and 0.897 (95\% CI: 0.863 to 0.923 ) and 0.863 (95\% CI: 0.831 to 0.889 ) among another 4,436 lesions from 4,097 patients in the 21 studies using supersonic shear imaging. When analysis confined to 9 studies evaluated the diagnostic performance of combination SWE and conventional ultrasound, the area under the curve was 0.96 (95\% CI: 0.94 to 0.97 ), yielding a sensitivity of 0.971 (95\% CI: 0.941 to 0.986 ) and specificity of 0.801 (95\% CI: 0.733 to 0.856 ). The authors concluded that SWE appeared to be a good quantitative method for differentiating breast lesions, with promise for integration into routine imaging protocols.

The results of this systematic review and metananlysis support the observation from prior studies that malignant lesions exhibit greater stiffnesses as measured by established mean and maximum metrics in SWE, whereas benign conditions represent a measurably softer population of masses. Large standard deviations in measurements from the papers included in this study suggest that this variability may contribute to the difficulty of discerning specific pathologies on the basis of mean or maximum elasticity alone.

In our study, subgroup analysis according to technology (VTTQ and SWE) was conducted. VTTQ showed higher detection specificity and accuracy than SWE. In terms of SWE parameters, the diagnostic performance of Emax was better than Emean.

\section{Study limitations:}

The meta-analysis was based on 8316 patients and 9057 breast lesions. The results were reliable and stable. However significant heterogeneity exhibited between the included studies. The heterogeneity might be caused by the patients' number, basic feature of patients, and experiments methods, and so on.

Elasticity measures are generally reported directly from the SWE system at the time of collection so post-processing of these quantities and subsequent variability due to this step is minimal. 
Prior studies that have qualitatively observed heterogeneity were however inconclusive relative to existing measures $[3,5,14]$.

Random error across all studies may even derive from elasticity changes in the tissues themselves. Fibroglandular tissue stiffness has been reported to change as much as $35 \%$ with menstrual cycle, in addition to morphologic changes. Menstrual and contraceptive hormones also affect the stiffness of the breast [24]. Perhaps the most significant changes in breast tissues occur with menopause [25], and menopausal status was not reported for any study reviewed. The use of biopsy is the accepted standard for diagnosing the lesion type, yet this is also a method of evaluation with known shortcomings and some inherent variability and misclassification [26].

\section{Conclusion:}

- Our meta-analysis demonstrates that SWE is an accurate and reliable diagnostic tool in discriminating malignant and benign breast lesions.

- With wide application, SWE may significantly improve the early diagnostic of breast cancer.

- In addition, SWE can provide additional information on predicting breast cancer prognosis. However, the possibility of false-positive and false-negative results should be considered during interpretation.

\section{References}

1- O'HAGAN J.J. and SAMANI A.: Measurement of the hyperelastic properties of 44 pathological ex vivo breast tissue samples. Physics in Medicine \& Biology, Apr. 6; 54 (8): 2557, 2009.

2- BARR R.G. and ZHANG Z.: Shear-wave elastography of the breast: Value of a quality measure and comparison with strain elastography. Radiology, 275: 45-53, 2015.

3- SADIGH G., CARLOS R.C., NEAL C.H. and DWAMENA B.A.: Accuracy of quantitative ultrasound elastography for differentiation of malignant and benign breast abnormalities: A meta-analysis. Breast cancer research and treatment. Aug. 1; 134 (3): 923-31, 2012.

4- ZHOU J., ZHAN W., CHANG C., ZHANG X., JIA Y., DONG Y., et al.: Breast lesions: Evaluation with shear wave elastography, with special emphasis on the "stiff rim" sign. Radiology, 272: 63-72, 2014.

5- BERG W.A., COSGROVE D.O., DORÉ C.J., SCHÄFER F.K., SVENSSON W.E., HOOLEY R.J., OHLINGER R., MENDELSON E.B., BALU-MAESTRO C., LOCATELLI M. and TOURASSE C.: Shear-wave elastography improves the specificity of breast US: The BE1 multinational study of 939 masses. Radiology. Feb., 262 (2): 435-49, 2012.

6- EVANS A., ARMSTRONG S., WHELEHAN P., THOMSON K., RAUCHHAUS P., PURDIE C., et al.: Can shear- wave elastography predict response to neoadjuvant chemotherapy in women with invasive breast cancer? Br. J. Cancer, 109: 2798-802, 2013

7- LEE S.H., CHANG J.M., KIM W.H., BAE M.S., CHO N., YI A., et al.: Differentiation of benign from malignant solid breast masses: Comparison of two-dimensional and three-dimensional shear-wave elastography. Eur. Radiol., 23: 1015-26, 2013.

8- BARR R.G. and ZHANG Z.: Effects of precompression on elasticity imaging of the breast: Development of a clinically useful semiquantitative method of precompression assessment. J. Ultrasound Med., 31: 895-902, 2012.

9- BURNSIDE E.S., HALL T.J., SOMMER A.M., et al.: Differentiating benign from malignant solid breast masses with US strain imaging. Radiology, 245: 401-10, 2007.

10- VREUGDENBURG T.D., WILLIS C.D., MUNDY L. and HILLER J.E.: A systematic review of elastography, electrical impedance scanning, and digital infrared thermography for breast cancer screening and diagnosis. Breast cancer research and treatment, Feb. 1; 137 (3): 665-76, 2013.

11- SAKORAFAS G.H., FARLEY D.R. and PEROS G.: Recent advances and current controversies in the management of DCIS of the breast. Cancer treatment reviews. Oct. 1; 34 (6): 483-97, 2008.

12- FERLAY J., SHIN H.R., BRAY F., et al.: Estimates of worldwide burden of cancer in 2008: GLOBOCAN 2008 Int. J. Cancer, 127: 2893-917, 2010.

13- BALLEYGUIER C., CIOLOVAN L., AMMARI S., et al.: Breast elastography: The technical process and its applications//Diagn Interv. Imaging, 94 (5)-P. 503-13, 2013.

14- BLANK M.A. and ANTAKI J.F.: Breast Lesion Elastography Region of Interest Selection and Quantitative Heterogeneity: A Systematic Review and MetaAnalysis // Ultrasound Med. Biol., Oct. 14. Pii: S0301-5629 (16) 30303-9, 2016.

15-ZHANG Q., XIAO Y., DAI W., et al.: Deep learning based classification of breast tumors with shear-wave elastography//Ultrasonics, 72. - P. 150-7, 2015.

16- CEBI D., KORKMAZER B., KILIC F., et al.: Use of shear wave elastography to differentiate benign and malignant breast lesions. Diagn. Interv. Radiol., 20: 23944, 2014.

17- YOUK J.H., GWEON H.M., SON E.J., et al.: Threedimensional shear-wave elastography for differentiating benign and malignant breast lesions: Comparison with two-dimensional shear-wave elastography. Eur. Radiol., 23: 1519-27, 2013.

18- KLOTZ T., BOUSSION V., KWIATKOWSKI F., et al.: Shear wave elastography contribution in ultrasound diagnosis management of breast lesions. Diagn. Interv. Imaging, 95: 813-24, 2014.

19- AU F.W., GHAI S., MOSHONOV H., et al.: Diagnostic performance of quantitative shear wave elastography in the evaluation of solid breast masses: Determination of the most discriminatory parameter. Am. J. Roentgenol., 203: W328-36, 2014.

20- NG W.L., RAHMAT K., FADZLI F., et al.: Shearwave Elastography Increases Diagnostic Accuracy in Charac- 
terization of Breast Lesions//Medicine (Baltimore), 95 (12): e3146, 2016.

21- CHUNG J., LEE W.K., CHA E.S., et al.: Shear-wave elastography for the differential diagnosis of breast papillary lesions. PloS One, 11 (11): e0167118, 2016.

22- CHEN L., HE J., LIU G., et al.: Diagnostic performances of shear-wave elastography for identification of malignant breast lesions: A meta-analysis. Jpn. J. Radiol., 32 (10): 592-9, 2014.

23- LIU B., ZHENG Y., HUANG G., et al.: Breast lesions: Quantitative diagnosis using ultrasound shear wave elastography-A systematic review and meta-analysis. Ultrasound Med. Biol., 42 (4): 835-47, 2016.
25- ELMORE J.G., LONGTON G.M., CARNEY P.A., GELLER B.M., ONEGA T., TOSTESON A.N.A., NELSON H.D., PEPE M.S., ALLISON K.H., SCHNITT S.J., MALLEY F.P.O. and WEAVER D.L.: Diagnostic concordance among pathologists interpreting breast biopsy specimens. JAMA, 313: 1122-32, 2015.

24- WOJCINSKI S., CASSEL M., FARROKH A., SOLIMAN A.A., HILLE U., SCHMIDT W., DEGENHARDT F. and HILLEMANNS P.: Variations in the elasticity of breast tissue during the menstrual cycle determined by real-time sonoelastography. J. Ultrasound Med., 31: 63-72, 2012.

26- GEFEN A. and DILMONEY B.: Mechanics of the normal woman's breast. Technol. Heal Care, 15: 259-71, 2007.

\section{التصوير المعتمد على المرونة بإستخلدام الموجات فوق الصوتية

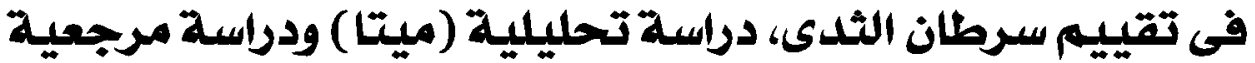

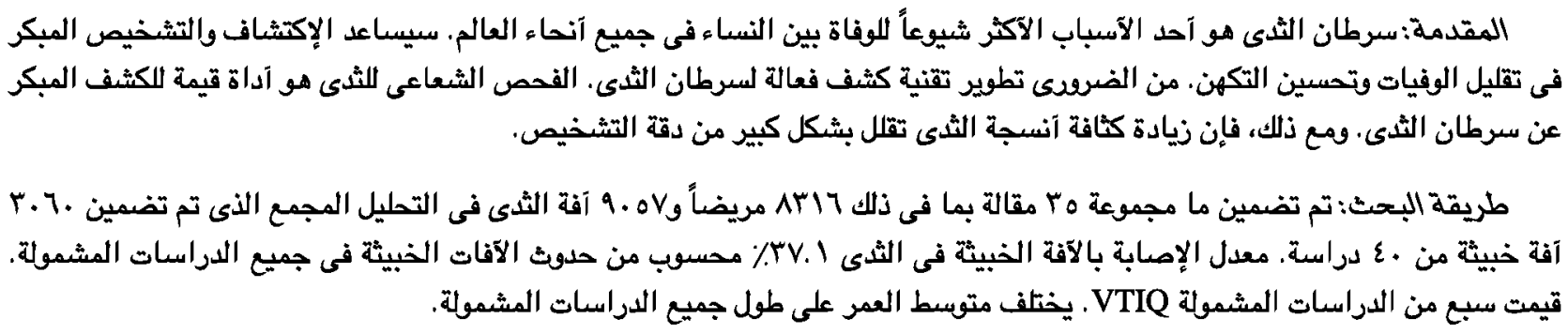

النتائج: إختلفت حساسية وخصوصية Emax gmean و التشائ Eratio لتشخيص سرطان الثدى وفقاً للمعايير التفسيرية المستخدمة لتعريف

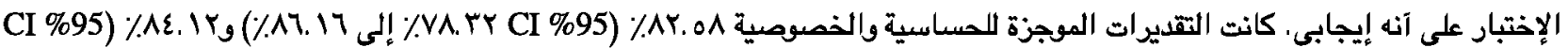

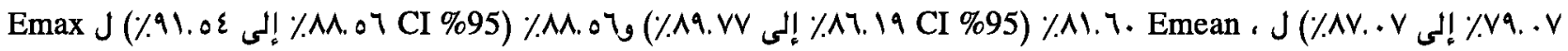

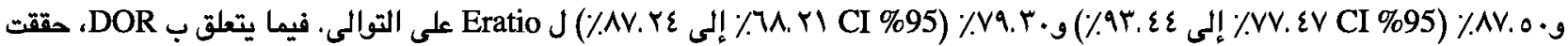

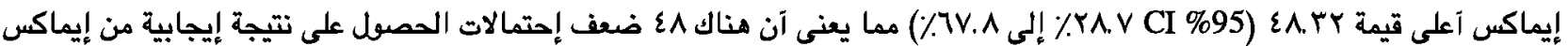

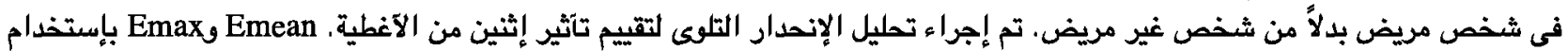

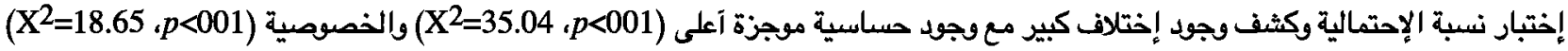
في Emax من Emean. تم إستخدام منحنيات SROC لإظهار توزيع حساسية وخصوصية Emax Emeang وEratio فى مساحة ROC وكتلك

منطقة التبنئ.

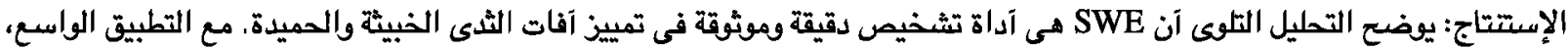

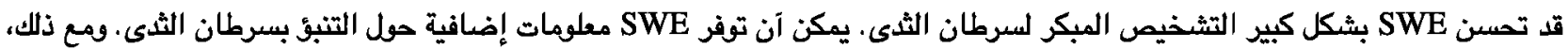

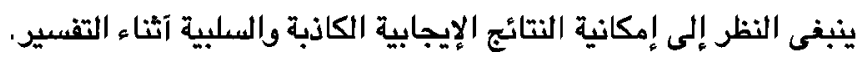

